\title{
Prognostic value of residual cancer burden and Miller-Payne system after neoadjuvant chemotherapy for breast cancer
}

\author{
Wei Wang ${ }^{1}$, Yinhua Liu ${ }^{1}$, Hong Zhang ${ }^{2}$, Shuang Zhang ${ }^{2}$, Xuening Duan ${ }^{1}$, Jingming $\mathrm{Ye}^{1}$, Ling Xu ${ }^{1}$, \\ Jianxin Zhao ${ }^{1}$, Yuanjia Cheng ${ }^{1}$, Qian Liu ${ }^{1}$ \\ ${ }^{1}$ Breast Disease Center, Peking University First Hospital, Beijing, China; ${ }^{2}$ Pathology Department, Peking University First Hospital, Beijing, China \\ Contributions: (I) Conception and design: W Wang, Y Liu, Q Liu; (II) Administrative support: H Zhang, S Zhang; (III) Provision of study materials \\ or patients: Y Liu, H Zhang, S Zhang, X Duan, J Ye, L Xu, J Zhao, Y Cheng; (IV) Collection and assembly of data: W Wang; (V) Data analysis and \\ interpretation: W Wang, Q Liu; (VI) Manuscript writing: All authors; (VII) Final approval of manuscript: All authors. \\ Correspondence to: Prof. Qian Liu. Peking University First Hospital, Beijing 100034, China. Email: 06744@pkufh.com.
}

\begin{abstract}
Backgroundk To verify the feasibility of using the residual cancer burden (RCB) index to stratify prognosis of patients after neoadjuvant chemotherapy (NAC) and to compare RCB with the Miller-Payne system.

Methods: We retrospectively analyzed clinicopathological data of patients receiving treatment between January 1, 2010 and December 31, 2018. Kaplan-Meier curves were used to compare the survival outcomes and estimate disease-free survival (DFS) and disease-specific survival (DSS). Harrell's concordance index (C-index) was used to evaluate the predictive accuracy of RCB and Miller-Payne system.

Results: A total of 423 female patients with complete data were included in the analysis, with a median follow-up time of 58.5 months (range, 7-126 months); 84 patients experienced recurrence, and 48 experienced breast cancer related death. RCB index and the Miller-Payne system were associated with prognosis in the whole cohort. Patients who achieved RCB-I had similar survival outcomes as those with pathological complete response (pCR, RCB-0). In whole cohort, for the RCB index and the Miller-Payne system, respectively, C-indexes for DFS were 0.73 and 0.64 , for DSS were 0.74 and 0.64 . The average RCB score was different among three subtypes $(\mathrm{F}=9.335, \mathrm{P}<0.001)$.

Conclusions: The RCB index and the Miller-Payne system can stratify survival outcome of patients after NAC, and RCB had a superior prediction accuracy, especially for triple-negative breast cancer (TNBC). New cut-off value should be sought in order to improve prediction accuracy.
\end{abstract}

Keywords: Breast cancer; neoadjuvant chemotherapy; residual cancer burden; Miller-Payne system; prognosis

Submitted Sep 02, 2021. Accepted for publication Nov 05, 2021.

doi: $10.21037 /$ gs-21-608

View this article at: https://dx.doi.org/10.21037/gs-21-608

\section{Introduction}

Neoadjuvant chemotherapy (NAC) is generally applied to locally advanced breast cancer. NAC can render inoperable tumors operable and can facilitate breast-conserving surgery by down-staging the tumor. The use of NAC may also provide important prognostic information based on treatment response.

Previous studies have shown that pathological complete response (pCR) is associated with improved survival outcomes, while patients with residual invasive carcinoma have poor prognoses (1). However, the dichotomy of pCR and non-pCR may be too simple, as the outcomes of patients who do not achieve pCR range from near pCR to frank resistance.

More than 15 neoadjuvant treatment pathology evaluation systems have been proposed to better stratify prognoses, including Miller-Payne system (2), American Joint Committee on Cancer (AJCC) staging system, residual cancer burden (RCB) (3), and Neo-Bioscore (4). The Miller-Payne system is currently the most commonly used pathology evaluation system in China, but it 
estimates only the reduction in primary tumor cellularity after treatment. The RCB index, however, utilizes calculations of the bidimensional diameter and cellularity of the primary tumor, and take lymph node metastasis into consideration, this system has been shown to measure the tumor burden more accurately, and its implementation is reproducible among pathologists $(5,6)$. Therefore, the $\mathrm{RCB}$ index is recommended by international working groups for measuring residual disease in clinical trials of NAC in the treatment of breast cancer $(7,8)$. So far, only a few small-scale retrospective studies verified the feasibility of the RCB index in the stratification of prognoses of patients treated with NAC (9-13), and none of them were based on the Chinese population. Thus, independent external validation of RCB is needed before it can be used as a surrogate endpoint in clinical trials.

In this study, we assessed the feasibility of using RCB to stratify prognoses of patients after NAC in a large single center in China, and we compared the results of the application of the RCB index to those obtained from the Miller-Payne system.

We present the following article in accordance with the STROBE reporting checklist (available at https://dx.doi. org/10.21037/gs-21-608).

\section{Methods}

\section{Patient population}

Female patients with primary invasive breast cancer treated with NAC between January 1, 2010 and December 31, 2018 were identified from a database created by Peking University First Hospital, Beijing, China. According to guidelines and previous study in our center, candidates for NAC in our center were those with: (I) human epidermal growth factor receptor 2 (HER2) positive disease or triple negative disease; (II) T3-4 tumors; (III) positive axillary lymph node; (IV) large primary tumor relative to breast size in a patient who desires breast conservation. The study entry criteria included: (I) women with unilateral invasive breast cancer diagnosed by core needle biopsy (CNB); (II) aged 18 to 75 years; (III) completed $\geq 4$ circles of neoadjuvant treatment; (IV) received surgical treatment; (V) necessary post-operative therapy, including endocrine therapy, anti-HER2 therapy, and radiotherapy. Patients were excluded in case of distant metastasis, with positive sentinel lymph node removed before NAC, occult breast cancer, or those without any follow-up record.

\section{Treatment strategy}

All patients underwent pathological biopsy of the primary tumor and suspected malignant axillary lymph nodes prior to initial treatment. The NAC regimen is based on taxane with or without anthracycline, and trastuzumab is applied to HER2+ patients. Magnetic resonance imaging examination was performed after every 2 cycles of therapy. In patients that exhibited tumor regression, the original treatment was continued; those with operable disease experiencing progression were proceed to surgery or given an alternate systemic therapy (14), which were generally based on vinorelbine in our center. All patients underwent breast-conserving surgery (BCS) or total mastectomy and axillary evaluation. Axillary lymph node dissection was performed for patients with positive CNB results. Patients with hormone receptor-positive disease routinely received adjuvant endocrine therapy for 5 to 10 years according to current guidelines, for premenopausal women tamoxifen \pm ovarian suppression or ablation was considered. The study period predated the use of pertuzumab in China, patients with HER2+ disease routinely completed only 1 year of trastuzumab treatment. Patients underwent BCS, and those with pathological $\mathrm{T}$ stage $\geq 3$, or pathological $\mathrm{N}$ stage $\geq 1$ routinely received standard postoperative radiotherapy.

\section{Collection of clinicopathological data}

Clinicopathologic data including age, presence of lymphovascular invasion, histological grade, tumor subtypes, clinical and pathological $\mathrm{T}, \mathrm{N}$ and $\mathrm{M}$ stages, pathological stages, chemotherapy regimen received, and RCB score. Hormone receptor positive was defined as estrogen receptors (ER) and/or progesterone receptors (PR) expression $>1 \%$. HER2 testing was performed according to 2013 guidelines of the American Society of Clinical Oncology/College of American Pathologists (ASCO/CAP) 2013, the positive criteria for HER2 were as follows: more than $10 \%$ of tumor cells with complete and intense membrane staining (by immunohistochemistry), or an average copy number $\geq 6$ signals/cell, or a dual-probe HER2/chromosome 17 centromere ratio $\geq 2$. Histological grade was evaluated according to Nottingham Combined Histologic Grade Scale. Clinical stage and pathological stage were determined according to the 8th AJCC Staging system.

Based on immunohistochemistry results, all cases were divided into three subtypes: luminal, positive for hormone 
receptor and negative for HER2; HER2-positive, positive for HER2 regardless of status of hormone receptor; triplenegative breast cancer (TNBC), negative for hormone receptor and HER2.

Miller-Payne system has 5 grades: grade 5 is a pathological complete response in breast; grades 1-4 are partial pathological response according to tumor reduction ratio (2). from $G 4$ to $G 1$, the degree of tumor reduction gradually decreases.

Based on the thresholds of 1.36 and 3.28, RCB index is divided into 4 classes: 0/pCR (ypT0/is, ypN0), minimal residual disease (RCB-I), moderate residual disease (RCBII), and extensive residual disease (RCB-III). RCB index was calculated by using web calculator published by MD Anderson (15).

\section{Follow-up procedures}

All patients received a regular postoperative follow-up, including physical examination and selected imaging examinations every 3-6 months during the first 3 years, and yearly thereafter. Data on follow-up were collected via investigation of the local electronic healthcare databases, outpatient, telephone interviews or letter once a year until April 30, 2021, patients without any postoperative information were considered lost to follow-up. The followup data include disease-free survival (DFS) and diseasespecific survival (DSS). DFS was defined as the time from definitive surgery to diagnosis of locoregional invasive disease or distant metastasis. Metachronous contralateral breast cancers were not considered as DFS events. DSS was defined as the time from definitive surgery to death related to breast cancer.

\section{Statistical analyses}

Descriptive statistics were used to describe characteristics of the patients. Kaplan-Meier survival curves were generated to compare the survival outcomes, intergroup comparison of DFS and DSS were performed by log-rank test. Hazard ratios (HRs) and their 95\% confidence intervals (CIs) were obtained through Cox models. Multivariable analyses were fit to determinate the association between RCB class and survival outcomes. One-way ANOVA was used to compare RCB index among three subtypes. Harrell's concordance index (C-index) was used to evaluate the predictive accuracy of RCB. Value of C-index near 0.5 indicates the model is completely random, while value of $\mathrm{C}$-index near 1 indicates the model is completely consistent with the fact (16). A z-score test using a one-shot nonparametric approach was used to compare two correlated C-index (17). Analyses were performed with $\mathrm{R}$ software (Version 4.0.3), and figures were produced using the package 'ggplot2' and 'compareC'. A significance level of 0.05 was used in all analyses.

\section{Ethical statement}

The study was conducted in accordance with the Declaration of Helsinki (as revised in 2013). The study was approved by the Institutional Ethics Examining Committee of Human Research of Peking University First Hospital (No. 2019-14) and informed consent was taken from all the patients.

\section{Results}

From January 2010 through December 2018, 538 female patients diagnosed with primary nonmetastatic invasive breast cancer received at least 4 cycles of NAC at the Breast Disease Center of Peking University First Hospital, China. 115 cases in this cohort were excluded: 13 who refused surgery in our center, 7 with simultaneous bilateral breast cancer, 44 with removal of a positive sentinel lymph node prior to NAC, 9 with occult breast cancer, 24 HER2 + patients who refused trastuzumab treatment, 11 with missing data and 7 who were lost during follow-up. Eventually, a total of 423 patients with complete data were included in the analysis.

The median age of this cohort was 50 years (range, $22-75$ years), with a median follow-up time of 58.5 months (range, 7-126 months). Invasive ductal carcinoma accounted for the majority of cases ( $n=373,88.2 \%)$. Approximately half of the cases involved grade 3 disease $(n=228,53.9 \%)$. Three subtypes each accounted for approximately onethird of the cases: 125 (29.6\%) patients were diagnosed with luminal breast cancer, 153 (36.1\%) patients were HER2+, and $145(34.3 \%)$ patients were diagnosed with TNBC. Of the enrolled patients, 355 (83.9\%) received an NAC regimen based on taxane with or without anthracycline, and $65(15.4 \%)$ patients received alternative chemotherapy based on vinorelbine and achieved good treatment response (Tables S1,S2). Most of the patients underwent mastectomy $(\mathrm{n}=320,75.7 \%)$ and axillary lymph node dissection (292, $69.0 \%)$. In our cohort, 101 (23.9\%) cases achieved pCR/ RCB-0, 74 (17.4\%) of the cases were staged as RCB-I, 161 (38.1\%) as RCB-II, and 87 (20.6\%) as RCB-III (Table 1). 


\section{Survival outcome}

During follow-up, 84 patients experienced recurrence, of which 12 were locoregional recurrences, and 5-year DFS rate was $81.7 \%$ (95\% CI: $77.9-85.7 \%$ ). There were 50 deaths, 48 were breast cancer related death. The 5 -year DSS rate was $88.5 \%$ (95\% CI: 85.2-91.9\%). Comparisons of DFS (Figure 1) and DSS (Figure 2) to grades determined by the RCB index and the Miller-Payne system are shown by Kaplan-Meier analyses. The estimated 5 -year survival rates and hazard ratios of patients in the various categories as calculated with the unadjusted Cox proportional hazards model are listed in Table 2.

In general, high RCB classes were associated with low rates of DFS and DSS and a high HR. All differences were statistically significant, except for DSS of the HER2+ subtype $(\mathrm{P}=0.4)$. Curves representing $0 / \mathrm{pCR}$ and RCB-I were adjacent in the global cohort and in all subtypes, and the differences were not statistically significant for DFS or DSS. In univariate (Table 2) and multivariate analyses (Table 3), the RCB class was confirmed as an independent prognostic factor for DFS and DSS, and there was no statistically significant difference in DFS or DSS between 0/pCR and RCB-I.

For the Miller-Payne system, the prognoses of patients who were evaluated as G5 were relatively good. The lower the $G$ grade, the worse the prognosis, this was true for both DFS and DSS, but the trend was not that obvious. Classifications using the Miller-Payne system were not significantly associated with DFS for the luminal subtype $(\mathrm{P}=0.14)$ or DSS for the HER2+ $(\mathrm{P}=0.16)$. Either DFS or DSS for luminal subtype was not completely positively correlated with the tumor remission rate. In the univariate analysis (Table 2), DFS rates were lower for G4 than for G5 (HR, 2.59; 95\% CI: 1.09-6.19; $\mathrm{P}=0.031$ ).

\section{Predictive accuracy of RCB and Miller-Payne systems}

In consideration of DFS, the C-index of the hierarchical RCB class was 0.73 when considering all patients in the cohort, 0.64 when considering patients with luminal type tumors, 0.66 when considering HER2+ patients and 0.83 when considering patients with TNBC. For DSS, the C-index was 0.74 for all patients, 0.72 for patients with luminal type tumors, 0.56 for HER2+ patients and 0.84 in patients with TNBC.

For DFS, the C-index for the Miller-Payne system was 0.64 globally, 0.53 when considering luminal type tumors,
0.62 in HER2 + tumors and 0.72 in patients with TNBC. For DSS, the C-index was 0.64 globally, 0.57 in luminal type tumors, 0.52 in HER2+ tumors and 0.72 in TNBC tumors.

In the whole cohort, luminal type tumors and TNBC, the $\mathrm{P}$ value for comparing two correlated $\mathrm{C}$-indices were all $<0.05$ based on DFS and DSS (Table 4), suggesting that the RCB was better than the Miller-Payne system.

\section{Differences of RCB among subgroups}

In the 322 cases with residual invasive tumors, the RCB score was higher in luminal type tumors compared with HER2+ tumors and TNBC tumors. The average RCB score was 2.73 (95\% CI: 2.52-2.93) in 115 luminal type tumors, 2.19 (95\% CI: 2.00-2.38) in 109 HER2 + tumors, and 2.39 (95\% CI: $2.27-2.50$ ) in 98 TNBC tumors. According a one-way ANOVA analysis, the differences were statistically significant $(\mathrm{F}=9.335 ; \mathrm{P}<0.0001)$.

\section{Discussion}

In this study, we verified the feasibility of using the RCB index and the Miller-Payne system to stratify patients treated with NAC based on survival outcomes. The RCB index showed superior performance relative to the MillerPayne system in the whole cohort and in each breast cancer subtype. Both systems had the best prediction accuracy in patients with TNBC. The relative accuracy in TNBC may be due to the fact that although TNBC can be further divided into different subtypes (18), the main treatment remains limited to chemotherapy, and the amount of residual tumor might therefore be the key factor that determines prognosis. In the other two subtypes, on the other hand, the expression of hormone and HER2 receptors are also associated with prognosis, which weakens the prognostic value of the tumor burden. We also noticed that, in both systems, the prediction accuracy for DSS in HER2+ patients was not much better than a simple coin flip. This was due to the small number of total events $(n=10)$, and 3 of these events occurred in patients who achieved 0/pCR, while 5 events occurred in patients who achieved grade G5. The Miller-Payne system ignores the evaluation of axillary lymph nodes, and will overestimate prognosis of patients with positive axillary lymph nodes. In our cohort, $63.2 \%$ (79/125) of patients of luminal subtype had involved axillary lymph nodes after NAC, which is much higher than the other two subtypes, and this may be an explanation for its 
Table 1 Clinicopathological characteristics of the whole cohort $(\mathrm{n}=423)$

\begin{tabular}{|c|c|}
\hline Variables & $\mathrm{N}=423(\%)$ \\
\hline Age (years), median [ range] & 50 [22-75] \\
\hline \multicolumn{2}{|l|}{ Menopausal status } \\
\hline Premenopausal & $214(50.6)$ \\
\hline Postmenopausal & $209(49.4)$ \\
\hline \multicolumn{2}{|l|}{ Tumor histological grade } \\
\hline 1 & $19(4.5)$ \\
\hline 2 & $176(41.6)$ \\
\hline 3 & $228(53.9)$ \\
\hline \multicolumn{2}{|l|}{ Subtypes } \\
\hline Luminal & $125(29.6)$ \\
\hline HER2+ & $153(36.1)$ \\
\hline TNBC & $145(34.3)$ \\
\hline \multicolumn{2}{|l|}{ Histology } \\
\hline Ductal & $373(88.2)$ \\
\hline Other & $39(9.2)$ \\
\hline Missing & $11(2.6)$ \\
\hline \multicolumn{2}{|l|}{ Lymphovascular invasion } \\
\hline Present & $65(15.4)$ \\
\hline Absent & $358(84.6)$ \\
\hline \multicolumn{2}{|l|}{ Chemotherapy regimen } \\
\hline Taxane \pm Anthracycline & $355(83.9)$ \\
\hline Taxane \pm Anthracycline + Vinorelbine & $65(15.4)$ \\
\hline Other & $3(0.7)$ \\
\hline \multicolumn{2}{|l|}{ Breast operation } \\
\hline BCS & $103(24.3)$ \\
\hline Mastectomy & $320(75.7)$ \\
\hline \multicolumn{2}{|l|}{ Axillary staging methods } \\
\hline SLNB & $131(31.0)$ \\
\hline ALND & $292(69.0)$ \\
\hline \multicolumn{2}{|l|}{ Clinical T category } \\
\hline $\mathrm{T} 1$ & $52(12.3)$ \\
\hline $\mathrm{T} 2$ & $316(74.7)$ \\
\hline T3 & $41(9.7)$ \\
\hline $\mathrm{T} 4$ & $14(3.3)$ \\
\hline \multicolumn{2}{|l|}{ Clinical $\mathrm{N}$ category } \\
\hline No & $148(35.0)$ \\
\hline $\mathrm{N} 1$ & $205(48.5)$ \\
\hline
\end{tabular}

Table 1 (continued)
Table 1 (continued)

\begin{tabular}{|c|c|}
\hline Variables & $\mathrm{N}=423(\%)$ \\
\hline N2 & $63(14.9)$ \\
\hline N3 & $7(1.6)$ \\
\hline \multicolumn{2}{|c|}{ Post-treatment Pathological T category } \\
\hline TO & $76(18.0)$ \\
\hline Tis & $40(9.5)$ \\
\hline $\mathrm{T} 1$ & $207(48.9)$ \\
\hline $\mathrm{T} 2$ & $77(18.2)$ \\
\hline T3 & $17(4.0)$ \\
\hline $\mathrm{T} 4$ & $6(1.4)$ \\
\hline \multicolumn{2}{|c|}{ Post-treatment Pathological N category } \\
\hline No & $249(58.9)$ \\
\hline $\mathrm{NO}(\mathrm{i}+)$ & $6(1.4)$ \\
\hline N1mi & $7(1.7)$ \\
\hline N1 & $79(18.7)$ \\
\hline N2 & $54(12.8)$ \\
\hline N3 & $28(6.6)$ \\
\hline \multicolumn{2}{|c|}{ Clinical stage } \\
\hline 1 & $20(4.7)$ \\
\hline II & $305(72.1)$ \\
\hline III & $98(23.2)$ \\
\hline \multicolumn{2}{|c|}{ Post-treatment pathological stage } \\
\hline 0 & $101(23.9)$ \\
\hline 1 & $134(31.7)$ \\
\hline II & $103(24.3)$ \\
\hline III & $85(20.1)$ \\
\hline \multicolumn{2}{|l|}{ RCB class } \\
\hline $0 / p C R$ & $101(23.9)$ \\
\hline 1 & $74(17.4)$ \\
\hline II & $161(38.1)$ \\
\hline III & $87(20.6)$ \\
\hline \multicolumn{2}{|c|}{ Evaluation of Miller-Payne system } \\
\hline G5 & $116(27.4)$ \\
\hline G4 & $88(20.8)$ \\
\hline G3 & $111(26.2)$ \\
\hline G2 & $37(8.8)$ \\
\hline G1 & $71(16.8)$ \\
\hline
\end{tabular}

HER2, human epidermal growth factor receptor 2; TNBC, triple negative breast cancer; BCS, breast-conserving surgery; SLNB, sentinel lymph node biopsy; ALND, axillary lymph node dissection; $\mathrm{RCB}$, residual cancer burden; $\mathrm{pCR}$, pathological complete response; $\mathrm{G}$, grade. 

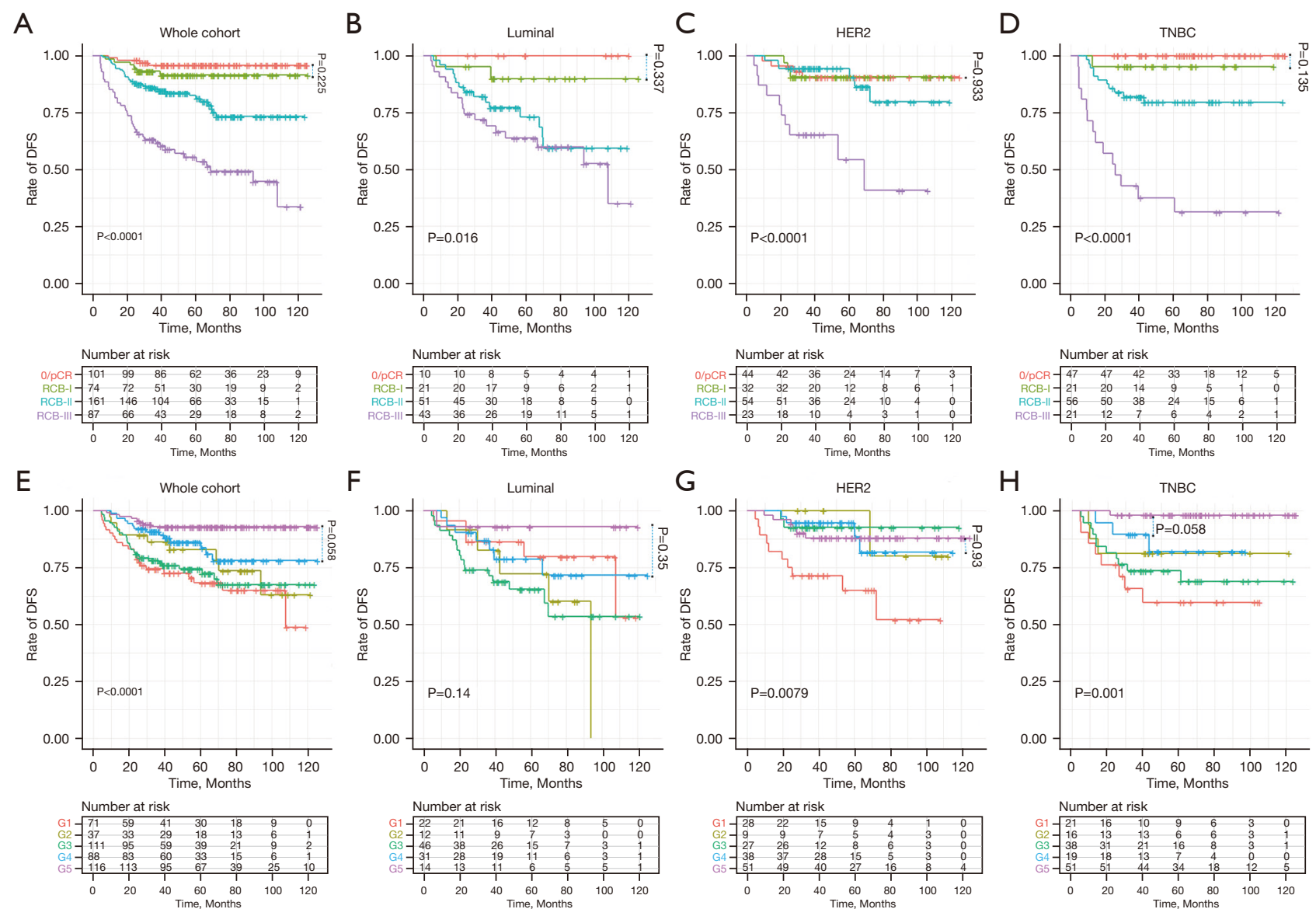

Figure 1 Kaplan-Meier survival curves of DFS according to RCB (A-D) and Miller-Payne (E-H) classification systems. DFS, disease-free survival; RCB, residual cancer burden.

poor performance in luminal subtype.

Prognostic stratification is also a valuable asset in the formulation of postoperative treatment plans. Our study suggested that patients achieved RCB-I have comparable survival outcomes as those who achieved 0/ pCR in the global cohort and within all subtypes; this finding was consistent with previous studies $(3,10,11)$. Based on the data accumulated in clinical trials, it seems that future improvements in NAC will more likely to occur when effective treatments are developed for specific patients with dismal prognosis. The most commonly used criterion to recruit patients into clinical trials is the lack of achievement of pCR after NAC. CREATE-X trial enrolled HER2 negative patients with residual disease (19), with 6-8 cycles of adjuvant capecitabine, the experimental group, especially the triple-negative patients, had better results. KATHERINE trial enrolled HER2positive patients with residual disease (20), 14 cycles of adjuvant trastuzumab emtansine did bring survival benefits to experimental group. Based on the results of these studies, the current guidelines for post-neoadjuvant treatment with residual disease for TNBC and HER2positive breast cancer, respectively, recommends intensive adjuvant chemotherapy. However, survival benefits brought by additional treatment were accompanied by an increase in adverse reactions, and a certain proportion of patients terminated treatment early or experienced dosage reductions. Based on the similarities between patients who achieved RCB-I and RCB-0, we propose that survival benefits in similar trials are limited for those enrolled patients with minimal residual disease (RCB-I). In order to increase the cost-effectiveness of medical resources and reduce unnecessary side effects caused by adjuvant treatment, future experiments are necessary in order to clarify whether these patients benefit from intensive adjuvant therapy. 

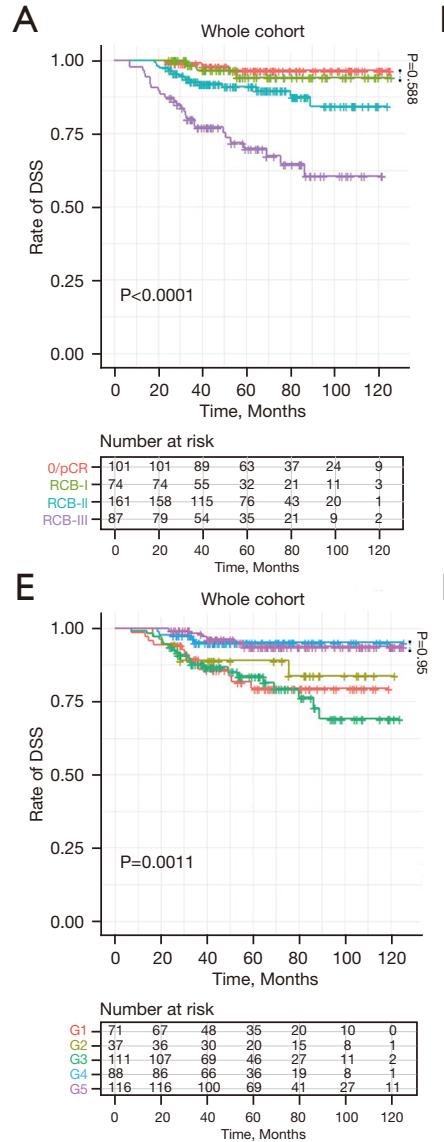

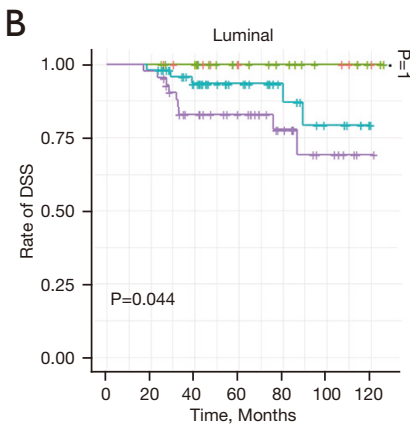

C
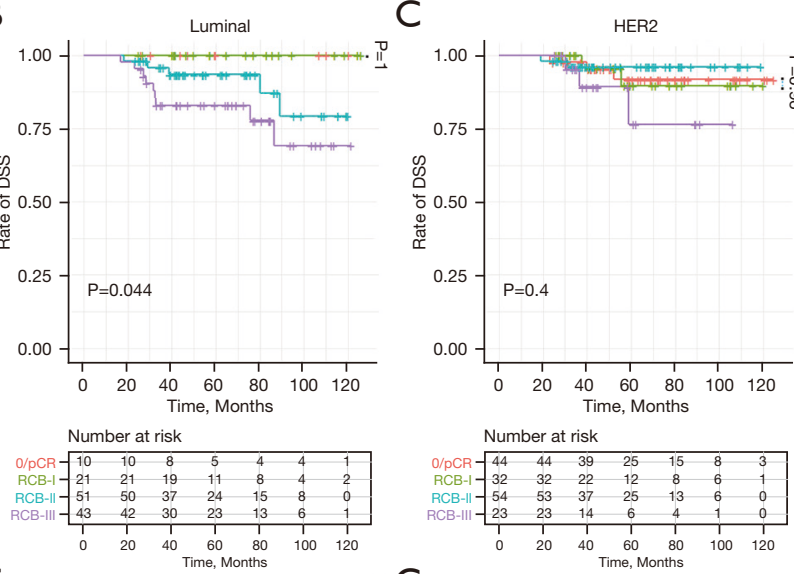

$\mathrm{F}$
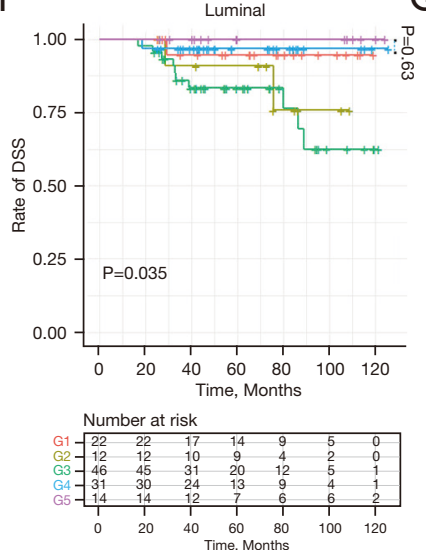

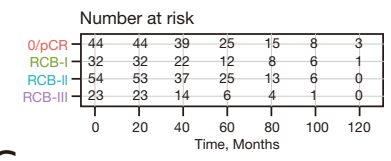

G
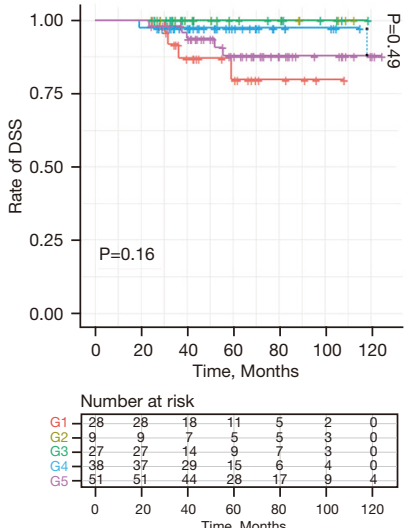
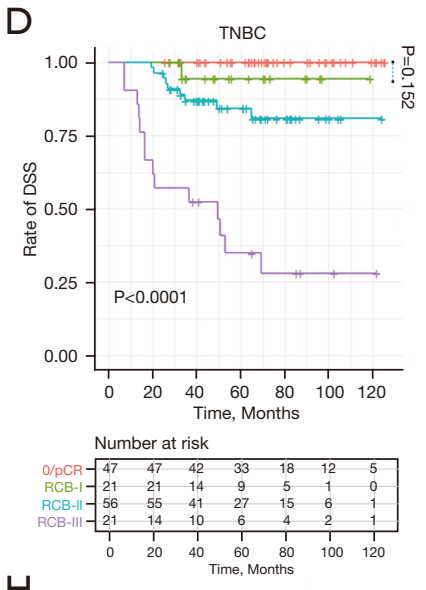

$\mathrm{H}$
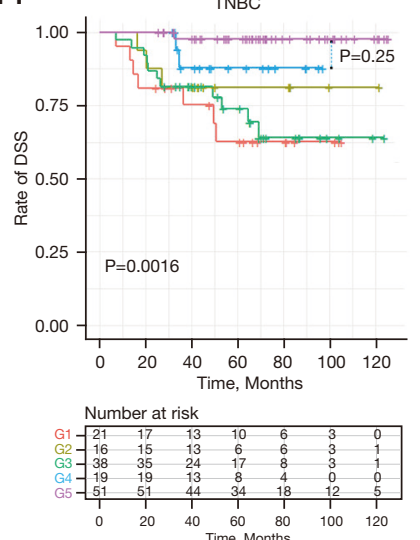

Figure 2 Kaplan-Meier survival curves of DSS according to RCB (A-D) and Miller-Payne (E-H) classification systems. DSS, disease-specific survival; RCB, residual cancer burden.

Table 2 Unadjusted 5 -year survival outcomes and hazard ratios by Cox regression analysis

\begin{tabular}{lcccccc}
\hline Staging system & Group & No & 5-year DFS $(\%)$ & HR $(95 \%$ Cl) & 5-year DSS (\%) & HR (95\% Cl) \\
\hline RCB class & O/pCR & 101 & 95.9 & Ref & 96.5 & Ref \\
& I & 74 & 91.4 & $2.28(0.64-8.09)^{\star}$ & 94.2 & $1.51(0.30-7.47)^{\star}$ \\
Miller-Payne system & II & 161 & 82.5 & $5.89(2.08-16.68)$ & 91.0 & $3.82(1.11-13.12)$ \\
& III & 87 & 55.5 & $16.97(6.08-47.37)$ & 69.9 & $12.68(3.82-41.72)$ \\
& G5 & 116 & 92.9 & Ref & 93.7 & Ref \\
& G4 & 88 & 86.2 & $2.59(1.09-6.19)$ & 95.1 & $0.99(0.28-3.52)^{\star}$ \\
& G3 & 111 & 74.4 & $4.81(2.21-10.52)$ & 83.6 & $4.32(1.74-10.73)$ \\
& G2 & 37 & 83.0 & $3.79(1.46-9.83)$ & 89.0 & $2.75(0.84-9.04)^{\star}$ \\
& G1 & 71 & 68.2 & $5.73(2.56-12.83)$ & 79.5 & $3.76(1.42-10.09)$ \\
\hline
\end{tabular}

*, P>0.05. Cl, confidence intervals; RCB, residual cancer burden; pCR, pathologic complete response; DFS, disease-free survival; DSS, disease-specific survival. 
Table 3 Multivariate analyses in the Cox proportional hazards model for DFS and DSS

\begin{tabular}{|c|c|c|c|c|c|c|}
\hline Variables & \multicolumn{3}{|c|}{ DFS } & \multicolumn{3}{|c|}{ DSS } \\
\hline Age (per 10 years increase) & 0.83 & $0.68-1.03$ & 0.086 & 0.93 & $0.716-1.22$ & 0.61 \\
\hline Clinical stage (III vs. I or II) & 1.46 & $0.90-2.35$ & 0.123 & 1.57 & $0.84-2.96$ & 0.160 \\
\hline \multicolumn{7}{|l|}{ Subtype } \\
\hline HER2+ & 0.90 & $0.52-1.55$ & 0.702 & 1.01 & $0.44-2.32$ & 0.973 \\
\hline TNBC & 1.14 & $0.68-1.92$ & 0.615 & 3.01 & $1.53-5.91$ & 0.001 \\
\hline LVI (present vs. absent) & 1.95 & $1.21-3.16$ & 0.006 & 1.84 & $0.96-3.52$ & 0.068 \\
\hline \multicolumn{7}{|l|}{ RCB class } \\
\hline II & 5.15 & $1.80-14.78$ & 0.002 & 3.98 & $1.14-13.84$ & 0.030 \\
\hline III & 13.05 & $4.50-37.90$ & $<0.0001$ & 12.31 & $3.47-43.69$ & $<0.0001$ \\
\hline
\end{tabular}

$\mathrm{CI}$, confidence intervals; DFS, disease-free survival; DSS, disease-specific survival; HER2, human epidermal growth factor receptor 2; TNBC, triple-negative breast cancer; LVI, lymphovascular Invasion; RCB, residual cancer burden, pCR, pathologic complete response.

Table 4 C-index of RCB class and Miller-Payne system for DFS and DSS

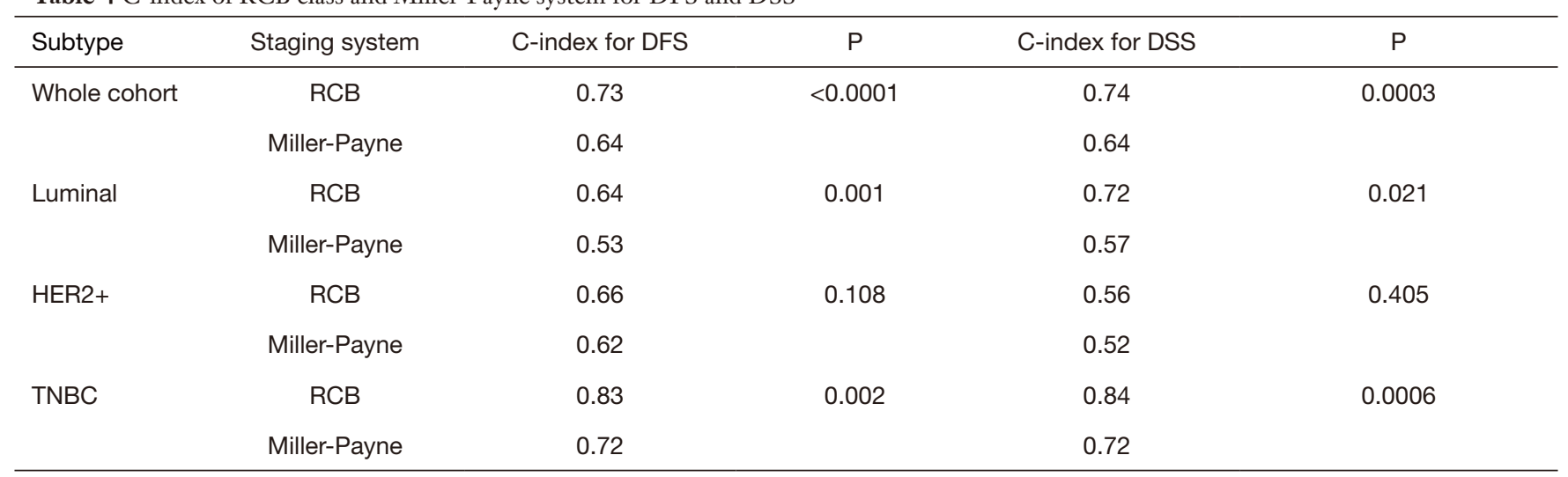

C-index, concordance-index; RCB, residual cancer burden; DFS, disease-free survival; DSS, disease-specific survival; HER2, human epidermal growth factor receptor 2; TNBC, triple negative breast cancer.

We observed that the average residual tumor burden was different among subgroups; therefore, a constant cutoff value might not apply globally to all patients. Furthermore, in the discovery cohort, all patients, including $20 \%(49 / 241)$ with HER2 + breast cancer, were treated with the same regimen of paclitaxel followed by fluorouracil, doxorubicin, and cyclophosphamide (T/FAC). In current practice, trastuzumab and pertuzumab combined with chemotherapy has become the standard treatment for HER2+ breast cancer, and this change in recommendation has led to significant improvements in tumor regression and survival outcomes (21). Therefore, the original cut-off value may no longer be appropriate, and it will be necessary to investigate further the appropriate subtype-specific cut-off values of RCB.

RCB remains limited to anatomical factors, and 
incorporating biological factors may further improve the performance of primary models. Sheri et al. showed that combining post-treatment analyses of Ki67 expression with RCB can improve the accuracy of predictions of survival outcomes (22), and this conclusion was subsequently validated in a small cohort (23). However, substantial variability of Ki67 staining of breast cancer between laboratories is a potential obstacle to the widespread clinical application of this strategy (24). Similarly, Laas et al. showed that addition of analyses of lymphovascular invasion and tumor-infiltrating lymphocytes to RCB can improve the predictive ability (9), but these two variables are not part of routine examinations in most pathology laboratories and are thus not likely to be widely applicable. One possible way to improve predictions is to use biological factors routinely reported by pathology laboratories, including grade and levels of expression of ER, PR and HER2, the feasibility of this strategy needs further study.

Our study had several limitations. First, as a retrospective study, a selection bias was inevitable. Second, our cohort was small, so any biases introduced by single outliers were magnified. Third, the follow-up time was relatively short, especially for luminal subtypes, which tend to recur after relatively long periods of time (25). With a prolonged time of follow-up, differences may show up in patients with the luminal subtype patients who achieved RCB-I and RCB-0 (26). A longer follow-up time, therefore, may result in better stratification results and accuracy of predictions based on RCB. We are currently attempting to enroll more patients in more centers to investigate the appropriate cut-off point for different subtypes with longer follow-up times.

In conclusion, our study verified that the RCB index and the Miller-Payne system can stratify survival outcomes of patients after NAC, but that RCB had better predictive accuracy, especially for TNBC. Patients who achieved RCB-I had similar prognoses to those with 0/pCR. Further studies should be devoted to the investigation of new cutoff values and to the incorporation of biological factors to improve predictive accuracy.

\section{Acknowledgments}

Funding: This work was supported by a grant from the Beijing Medical Award Foundation (No. 2018-0304).

\section{Footnote}

Reporting Checklist: The authors have completed the STROBE reporting checklist. Available at https://dx.doi. org/10.21037/gs-21-608

Data Sharing Statement: available at https://dx.doi. org/10.21037/gs-21-608

Peer Review File: Available at https://dx.doi.org/10.21037/ gs-21-608

Conflicts of Interest: All authors have completed the ICMJE uniform disclosure form (available at https://dx.doi. org/10.21037/gs-21-608). The authors have no conflicts of interest to declare.

Ethical Statement: The authors are accountable for all aspects of the work in ensuring that questions related to the accuracy or integrity of any part of the work are appropriately investigated and resolved. The study was conducted in accordance with the Declaration of Helsinki (as revised in 2013). The study was approved by the Institutional Ethics Examining Committee of Human Research of Peking University First Hospital (No. 2019-14) and informed consent was taken from all the patients.

Open Access Statement: This is an Open Access article distributed in accordance with the Creative Commons Attribution-NonCommercial-NoDerivs 4.0 International License (CC BY-NC-ND 4.0), which permits the noncommercial replication and distribution of the article with the strict proviso that no changes or edits are made and the original work is properly cited (including links to both the formal publication through the relevant DOI and the license). See: https://creativecommons.org/licenses/by-ncnd/4.0/.

\section{References}

1. Cortazar P, Zhang L, Untch M, et al. Pathological complete response and long-term clinical benefit in breast cancer: the CTNeoBC pooled analysis. Lancet 2014;384:164-72.

2. Ogston KN, Miller ID, Payne S, et al. A new histological grading system to assess response of breast cancers to 
primary chemotherapy: prognostic significance and survival. Breast 2003;12:320-7.

3. Symmans WF, Peintinger F, Hatzis C, et al.

Measurement of residual breast cancer burden to predict survival after neoadjuvant chemotherapy. J Clin Oncol 2007;25:4414-22.

4. Mittendorf EA, Vila J, Tucker SL, et al. The NeoBioscore Update for Staging Breast Cancer Treated With Neoadjuvant Chemotherapy: Incorporation of Prognostic Biologic Factors Into Staging After Treatment. JAMA Oncol 2016;2:929-36.

5. Peintinger F, Sinn B, Hatzis C, et al. Reproducibility of residual cancer burden for prognostic assessment of breast cancer after neoadjuvant chemotherapy. Mod Pathol 2015;28:913-20.

6. Naidoo K, Parham DM, Pinder SE. An audit of residual cancer burden reproducibility in a UK context. Histopathology 2017;70:217-22.

7. Provenzano E, Bossuyt V, Viale G, et al. Standardization of pathologic evaluation and reporting of postneoadjuvant specimens in clinical trials of breast cancer: recommendations from an international working group. Mod Pathol 2015;28:1185-201.

8. Bossuyt V, Provenzano E, Symmans WF, et al. Recommendations for standardized pathological characterization of residual disease for neoadjuvant clinical trials of breast cancer by the BIG-NABCG collaboration. Ann Oncol 2015;26:1280-91.

9. Laas E, Labrosse J, Hamy AS, et al. Determination of breast cancer prognosis after neoadjuvant chemotherapy: comparison of Residual Cancer Burden (RCB) and NeoBioscore. Br J Cancer 2021;124:1421-7.

10. Kantor O, Laws A, Pastorello RG, et al. Comparison of Breast Cancer Staging Systems After Neoadjuvant Chemotherapy. Ann Surg Oncol 2021;28:7347-55.

11. Müller HD, Posch F, Suppan C, et al. Validation of Residual Cancer Burden as Prognostic Factor for Breast Cancer Patients After Neoadjuvant Therapy. Ann Surg Oncol 2019;26:4274-83.

12. Campbell JI, Yau C, Krass P, et al. Comparison of residual cancer burden, American Joint Committee on Cancer staging and pathologic complete response in breast cancer after neoadjuvant chemotherapy: results from the I-SPY 1 TRIAL (CALGB 150007/150012; ACRIN 6657). Breast Cancer Res Treat 2017;165:181-91.

13. Choi M, Park YH, Ahn JS, et al. Assessment of pathologic response and long-term outcome in locally advanced breast cancers after neoadjuvant chemotherapy: comparison of pathologic classification systems. Breast Cancer Res Treat 2016;160:475-89.

14. NCCN Clinical Practice Guidelines in Oncology-Breast Cancer (2021 Version 1)[DB/OL]. Available online: http:// www.nccn.org.

15. The University of Texas, MD Anderson Cancer Center Residual Cancer Burden Calculator. Available online: www.mdanderson.org/breastcancer_RCB

16. Harrell FE Jr, Califf RM, Pryor DB, et al. Evaluating the yield of medical tests. JAMA 1982;247:2543-6.

17. Kang L, Chen W, Petrick NA, et al. Comparing two correlated $\mathrm{C}$ indices with right-censored survival outcome: a one-shot nonparametric approach. Stat Med 2015;34:685-703.

18. Jiang YZ, Ma D, Suo C, et al. Genomic and Transcriptomic Landscape of Triple-Negative Breast Cancers: Subtypes and Treatment Strategies. Cancer Cell 2019;35:428-440.e5.

19. Masuda N, Lee SJ, Ohtani S, et al. Adjuvant Capecitabine for Breast Cancer after Preoperative Chemotherapy. N Engl J Med 2017;376:2147-59.

20. von Minckwitz G, Huang CS, Mano MS, et al. Trastuzumab Emtansine for Residual Invasive HER2Positive Breast Cancer. N Engl J Med 2019;380:617-28.

21. Gianni L, Pienkowski T, Im YH, et al. 5-year analysis of neoadjuvant pertuzumab and trastuzumab in patients with locally advanced, inflammatory, or earlystage HER2-positive breast cancer (NeoSphere): a multicentre, open-label, phase 2 randomised trial. Lancet Oncol 2016;17:791-800.

22. Sheri A, Smith IE, Johnston SR, et al. Residual proliferative cancer burden to predict long-term outcome following neoadjuvant chemotherapy. Ann Oncol 2015;26:75-80.

23. Miglietta F, Dieci MV, Tsvetkova V, et al. Validation of Residual Proliferative Cancer Burden as a Predictor of Long-Term Outcome Following Neoadjuvant Chemotherapy in Patients with Hormone ReceptorPositive/Human Epidermal Growth Receptor 2-Negative Breast Cancer. Oncologist 2020;25:e1355-62.

24. Focke CM, Bürger H, van Diest PJ, et al. Interlaboratory variability of Ki67 staining in breast cancer. Eur J Cancer 2017;84:219-27.

25. Prat A, Pineda E, Adamo B, et al. Clinical implications of the intrinsic molecular subtypes of breast cancer. Breast 
2015;24 Suppl 2:S26-35.

26. Symmans WF, Wei C, Gould R, et al. Long-Term Prognostic Risk After Neoadjuvant Chemotherapy

Cite this article as: Wang W, Liu Y, Zhang H, Zhang S, Duan X, Ye J, Xu L, Zhao J, Cheng Y, Liu Q. Prognostic value of residual cancer burden and Miller-Payne system after neoadjuvant chemotherapy for breast cancer. Gland Surg 2021;10(12):3211-3221. doi: 10.21037/gs-21-608
Associated With Residual Cancer Burden and Breast Cancer Subtype. J Clin Oncol 2017;35:1049-60. 
Supplementary

Table S1 Use the Miller-Payne system to measure the tumor burden after alternative chemotherapy based on vinorelbine

\begin{tabular}{lcccc}
\hline & Whole cohort & Luminal & HER2 & TNBC \\
\hline Total & 65 & 18 & 15 & 72 \\
G1 & 18 & 4 & 2 & 7 \\
G2 & 11 & 2 & 4 & 9 \\
G3 & 21 & 8 & 2 & 0 \\
G4 & 5 & 3 & 0 & 9 \\
G5 & 10 & 1 & 9 & 9 \\
\hline
\end{tabular}

HER2, human epidermal growth factor receptor 2.

Table S2 Use the residual cancer burden to measure tumor burden after alternative chemotherapy based on vinorelbine

\begin{tabular}{lcccc}
\hline & Whole cohort & Luminal & HER2 & TNBC \\
\hline Total & 65 & 18 & 15 & 32 \\
O/RCB-0 & 9 & 1 & 2 & 1 \\
RCB-I & 4 & 1 & 5 & 14 \\
RCB-II & 28 & 9 & 8 & 9 \\
RCB-III & 24 & 7 & 8 & 8 \\
\hline
\end{tabular}

HER2, human epidermal growth factor receptor 2. 